Check for updates

Cite this: RSC Adv., 2019, 9, 37825

Received 4th September 2019

Accepted 25th October 2019

DOI: 10.1039/c9ra08709e

rsc.li/rsc-advances

\section{Platinum-(phosphinito-phosphinous acid) complexes as bi-talented catalysts for oxidative fragmentation of piperidinols: an entry to primary amines $\uparrow$}

\author{
Romain Membrat, ${ }^{a}$ Alexandre Vasseur, (D) ${ }^{\mathrm{b}}$ Delphine Moraleda, ${ }^{\mathrm{a}}$ Sabine Michaud- \\ Chevallier, ${ }^{a}$ Alexandre Martinez, (D) ${ }^{* a}$ Laurent Giordano*a and Didier Nuel (DD *a
}

\section{Introduction}

The terminology "metal-catalyzed hydrogen transfer methodology" refers to any chemical process relying on the generation of a metal hydride from a hydrogen source and its transfer to an unsaturated hydrogen acceptor. ${ }^{1,2}$ A substantial number of consistent versions of this concept have been reported and implemented with palladium catalysts for alcohols oxidation, ${ }^{3-5}$ hydrogenation, ${ }^{6,7} \mathrm{C}-\mathrm{C},{ }^{8,9} \mathrm{C}-\mathrm{N},{ }^{10} \mathrm{C}$-halogen bond formation, ${ }^{11}$ double bond isomerization, ${ }^{12}$ cross aldolisation ${ }^{13}$ or else amination ${ }^{14}$ with a view to increasing the molecular complexity. The critical feature of metal-catalyzed hydrogen transfer methodology lays in the design of suitable ligands, able to induce the formation of a reactive metal hydride intermediate. ${ }^{4,7,15}$ In this context, we recently reported that palladium and platinum phosphinous acid (PA) complexes can generate active metal hydrides. ${ }^{16}$ This property is due to the self-assembled hydrogen bond negatively charged assisted structure of two PA ligands (M/PAP complexes, Scheme 1). ${ }^{17}$ These catalysts allowed therefore the anaerobic oxidation of notably reluctant alcohols such as $N$-alkyl-(2,2,6,6)-tetramethylpiperidin-4-ols $\mathbf{1 .}{ }^{18}$ In earlier years, the M/PAP structure proved to be also active as a Lewis acid toward sterically congested substrates in tandem $[2+1]$ cycloaddition-ring expansion of norbornene derivatives with propargylic acetates. ${ }^{19}$ In order to benefit from this noteworthy M/PAP's multiskilling ${ }^{20}$ we focused our effort on a new hydrogen transfer induced transformation where the catalyst acts as both

${ }^{a}$ Aix-Marseille Univ, CNRS, Centrale Marseille, iSm2, Marseille, France. E-mail: didier. nuel@centrale-marseille.fr

${ }^{b}$ Université de Lorraine, CNRS, L2CM, F-54000 Nancy, France

$\dagger$ Electronic supplementary information (ESI) available. See DOI: 10.1039/c9ra08709e a hydrogen carrier and a Lewis acid in a single chemical system (Scheme 1(a)).

On one hand, we recently reported the Pt(II)-SPO complexcatalyzed oxidation the $N$-alkyl-2,2,6,6-tetramethylpiperidin4-ols into their corresponding piperidinone derivatives. ${ }^{18}$ On the other hand $N$-alkyl piperidinone motif have been reported earlier as a source of $N$-alkyl primary amines. ${ }^{21-26}$ We hypothesized that the electrophilic features of bisphosphinite metallacycle would also promote the double $\mathrm{C}\left(\mathrm{sp}^{3}\right)-\mathrm{N}$ bond cleavage. Combining these two steps with a single catalyst the whole process would emerge as a readily access to primary amines (Scheme 1b). Herein we report our results in this regard.

\section{Results and discussion}

During our studies on the palladium catalyzed anaerobic oxidation of $\mathrm{N}$-alkyl-2,2,6,6-tetramethylpiperidin-4-ols ${ }^{18}$ derivatives, we observed, in the case of $1 \mathbf{a}$, that product $2 \mathrm{a}$ was found only in trace amount along with phorone (2,6-dimethylhepta2,5-dien-4-one) 3, which was isolated in 90\% yield (Scheme 2).

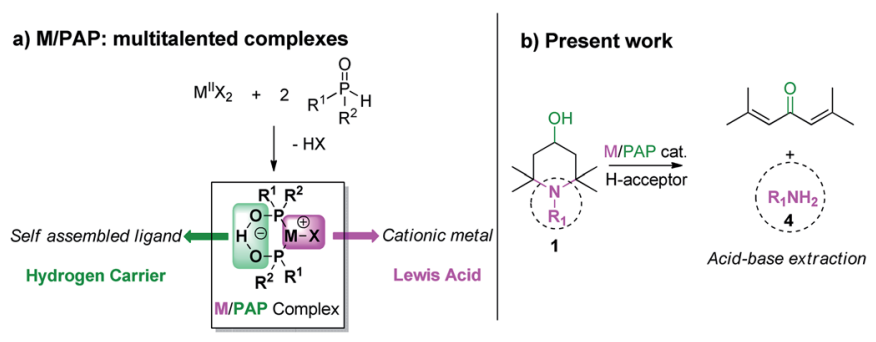

Scheme 1 M/PAP complexes as bitalented catalyst. 


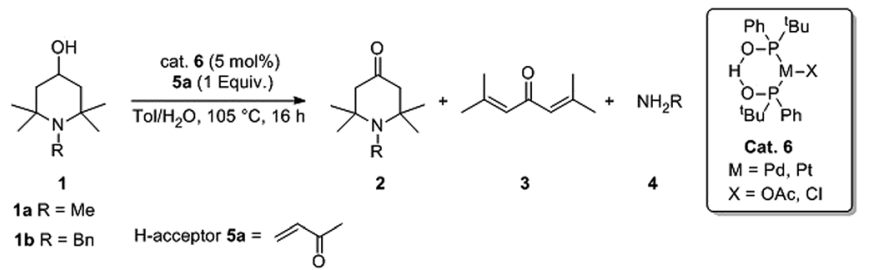

Scheme 2 M/PAP complex catalyzed oxidative fragmentation of piperidinols.

We hypothesise that formation of $\mathbf{3}$ arose through the cleavage of $\mathrm{C}-\mathrm{N}$ bonds in the piperidinone $2 \mathrm{a}$ leading to methylamine 4a (not isolated). According to this result, the development of a one pot oxidative fragmentation of piperidinols 1 to deliver primary amines 4 should be possible (Scheme 1(b)). Thus, a preliminary study was carried out using $N$-benzyl-2,2,6,6-tetramethylpiperidin-4-ol $\mathbf{1 b}$ in order to isolate benzylamine $\mathbf{4 b}$ (Scheme 2 ).

The reaction of $\mathbf{1 b}$ and electron-poor alkene $\mathbf{5 a}$ in the presence of $5 \mathrm{~mol} \%$ of Pd/PAP or Pt/PAP complexes 6 in toluene/ water $(9 / 1)$ at $105{ }^{\circ} \mathrm{C}$ was examined (Table 1$)$.

The experiment conducted in 4 hours with the catalyst 6 a proved disappointing as the conversion was incomplete and only $14 \%$ of phorone 3 were detected (Table 1 , entry 1 ). Increasing the reaction time led to complete conversion but the ring opening remained incomplete affording a mixture of $2 \mathbf{b}: \mathbf{3}$ in a $70: 30$ ratio (Table 1 , entry 2 ). Catalyst $6 \mathbf{b}$ also led to complete conversion with a decreased yield of phorone 3 (Table 1 , entry 3). The best ratio of $2 \mathbf{b}: 3$ (64:36) was observed with the platinum complex $6 \mathrm{c}$ and improved to $40: 60$ with a longer reaction time (Table 1, entries 4 and 5) suggesting a sequential oxidation-opening process. In a previous work, we showed that treatment of $\mathbf{6 c}$ with $\mathrm{NaOH}$ afforded a hydroxy-platinum catalyst 6d as active species (see Table 2) for the chemoselective anaerobic oxidation of $\mathbf{1 b}$ with $\mathbf{5 a}$ as $\mathrm{H}$-acceptor and allowed the oxidation to occur at room temperature. We decided then to try the reaction in these conditions to obtain the ketone prior to optimize the ring opening step (Table 2).

Table 1 Feasibility of the oxidation/C-N bond cleavage tandem sequence of $1 b^{a}$

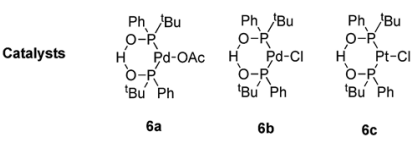

\begin{tabular}{llll}
\hline Entry & Cat. & $t(\mathrm{~h})$ & $\mathbf{1 b}: \mathbf{2 b}: \mathbf{3}$ ratio $^{b}(\%)$ \\
\hline 1 & $\mathbf{6 a}$ & 4 & $14: 71: 14$ \\
2 & $\mathbf{6 a}$ & 16 & $0: 70: 30$ \\
3 & $\mathbf{6 b}$ & 16 & $0: 78: 22$ \\
4 & $\mathbf{6 c}$ & 16 & $0: 64: 36$ \\
5 & $\mathbf{6 c}$ & 72 & $0: 40: 60$
\end{tabular}

${ }^{a}$ All reactions were carried out with $1 \mathrm{mmol}$ of $1 \mathbf{b}$ in toluene $/ \mathrm{H}_{2} \mathrm{O} 9 / 1$ at $105{ }^{\circ} \mathrm{C} .1 \mathrm{~b} / 5 \mathrm{a} /$ cat. $6=1 / 2 / 0.05 .{ }^{b}$ Determined by ${ }^{1} \mathrm{H}$ NMR spectroscopy.
Under these conditions, the oxidation of $\mathbf{1 b}$ to $\mathbf{2} \mathbf{b}$ was complete in 4 hours and the amine $\mathbf{4 b}$ was not detected even under prolonged reaction time like 16 hours at $105{ }^{\circ} \mathrm{C}$ (Table 2 , entry 1 ). In this case, we supposed that the relative basicity of the medium precludes the cleavage process of $\mathbf{2} \mathbf{b}$. Indeed, in the reaction conditions described in Table 1 (with complexes 6a, 6b and $\mathbf{6 c}$ ) no base was added, and catalytic traces of $\mathrm{HCl}$ or $\mathrm{AcOH}$ are probably released during the formation of the $[\mathrm{M}]-\mathrm{OH}$ active species, which probably promotes the cleavage of $\mathbf{2 b}$. Thus, once the oxidation reaction under basic conditions is over (typically after 4 hours), 1 equivalent of $\mathrm{HCl}$ or $\mathrm{AcOH}$ (Table 2, entries 2 and 4) was added directly to the mixture.

Results comparable to the reactions done in neutral medium using $\mathbf{6 a}, \mathbf{6 b}$ or $\mathbf{6 c}$ as catalyst (Table 1 , entries 2-4) were obtained. However, an excess of $\mathrm{HCl}$ ( 5 equiv.) resulted in a complete deactivation of the catalyst (Table 2 , entry 3 ). In contrast, the use of an excess of acetic acid ( 5 equiv.) (Table 2 , entry 5) was shown to be very beneficial as quantitative deprotection of piperidinone $\mathbf{2 b}$ was observed after $16 \mathrm{~h}$ at $105{ }^{\circ} \mathrm{C}$. Unfortunately only traces of free amine $\mathbf{4 b}$ were detected probably due to the possible 1,4-addition of the amine to the MVK 5a (Table 2, entries 2, 4 and 5). ${ }^{27}$ We then seek for a less reactive hydrogen acceptor to the addition of amine. Trans-phenyl-but-3-en-2-one $\mathbf{5 b}$ was finally selected as the $\mathrm{H}$-acceptor as it gives a clean total conversion and $\mathbf{4 b}$ was obtained in a pure form in $88 \%$ isolated yield by simple acidbase extraction without further purification (Table 2, entry 6). The optimal conditions for the reaction were refined to $105{ }^{\circ} \mathrm{C}$ during 4 hours for the first step and 5 hours for the second (Table 2, entry 7).

Table 2 Optimization of the sequence ${ }^{a}$

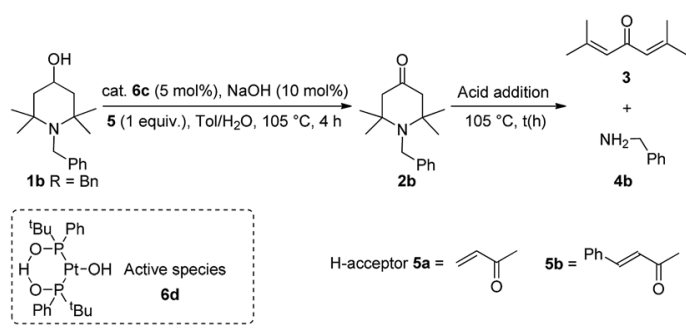

\begin{tabular}{lllll} 
Entry & H-acceptor & Acid (equiv.) & $t(\mathrm{~h})$ & $\mathbf{2 b}: \mathbf{3}: \mathbf{4} \mathbf{b}$ ratio $^{b}(\%)$ \\
\hline 1 & $\mathbf{5 a}$ & - & 16 & $100: 0: 0$ \\
2 & $\mathbf{5 a}$ & $\mathrm{HCl}(1)$ & 16 & $82: 18: \mathrm{ND}^{c}$ \\
3 & $\mathbf{5 a}$ & $\mathrm{HCl}(5)$ & 16 & $100: 0: 0$ \\
4 & $\mathbf{5 a}$ & AcOH (1) & 16 & $72: 28: \mathrm{ND}^{c}$ \\
5 & $\mathbf{5 a}$ & AcOH (5) & 16 & $0: 100: \mathrm{ND}^{c}$ \\
6 & $\mathbf{5 b}$ & AcOH (5) & 16 & $0: 100: 100$ \\
7 & $\mathbf{5 b}$ & AcOH (5) & 5 & $0: 100: 100$
\end{tabular}

${ }^{a}$ All reactions were carried out with $1 \mathrm{mmol}$ of $\mathbf{1 b}$ in toluene $/ \mathrm{H}_{2} \mathrm{O} 9 / 1$ at $105{ }^{\circ} \mathrm{C}, 4 \mathrm{~h} . \mathbf{1} / 5 \mathbf{b} / 6 \mathbf{c}=1 / 2 / 0.05$ and then addition of acid ( 5 equiv.), $105{ }^{\circ} \mathrm{C} .{ }^{b}$ Determined by ${ }^{1} \mathrm{H}$ NMR spectroscopy. ${ }^{c} \mathbf{4 b}$ is detected in trace amounts. 


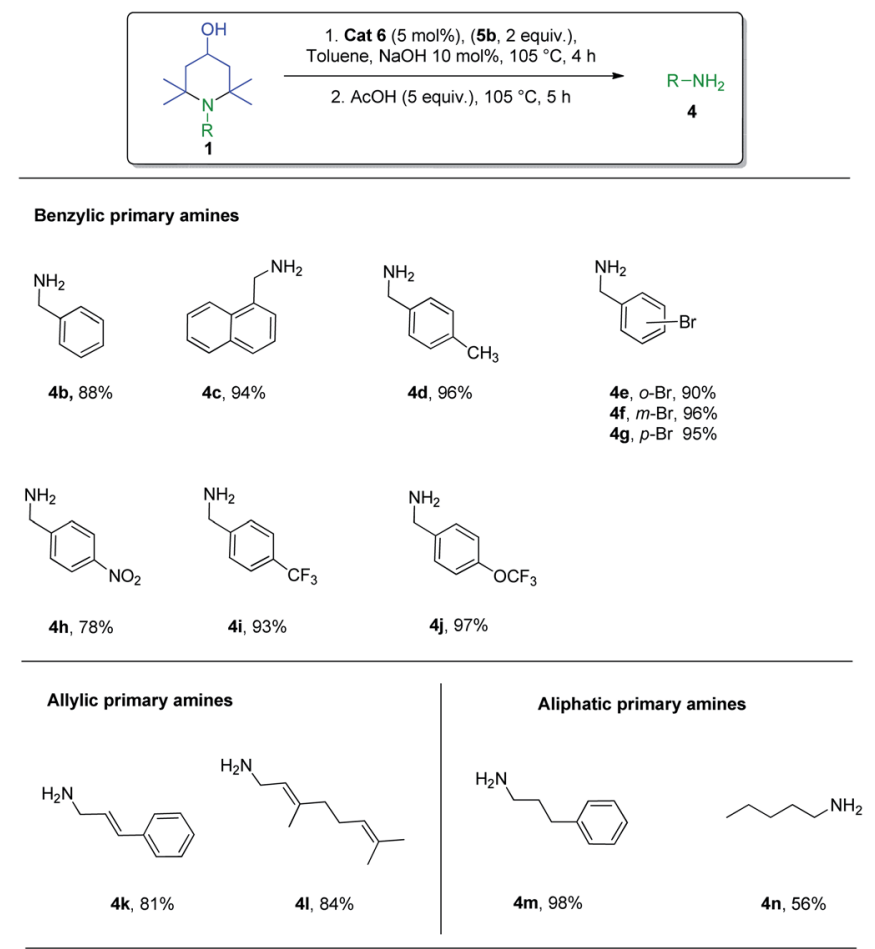

Functionalized and congested primary amines

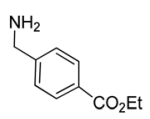

$40,71 \%$

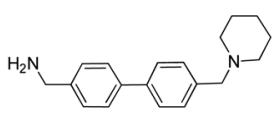

4p, $90 \%$

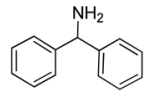

4q, $38 \%$
Scheme 3 Scope of the process.

Having established the feasibility of the oxidation-opening sequence, we synthesized various $N$-alkyl-2,2,6,6tetramethylpiperidin-4-ols $\mathbf{1} \mathbf{b}-\mathbf{q}$ to extend its applicability. ${ }^{28,29}$ Under optimized conditions, the conversion of $\mathbf{1}$ proceeded cleanly and the amines $\mathbf{4}$ were obtained in good to excellent yields without chromatography (Scheme 3). All benzylic amines were obtained in very good yields whatever is the substitution on the phenyl ring (Scheme 3, $\mathbf{4 b} \mathbf{b} \mathbf{4 j}$ ). The electronic features of the functional groups within the phenyl moiety have no major influence on the outcome of the reaction (compare for instance 4d with 4i). Allylic amines are also efficiently produced (compounds $\mathbf{4 k}$ and $\mathbf{4 l})$ as well as aliphatic amines $(\mathbf{4 m}, \mathbf{4 n})$. The obtention of product $\mathbf{4 p}$ stressed the already presumed necessity of the activation of the protective group by a ketone function. Even a congested piperidinol (1q) was able to deliver

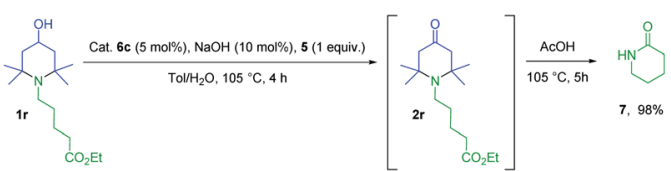

Scheme 4 Application to three steps-one pot oxidation-fragmentation-cyclization.

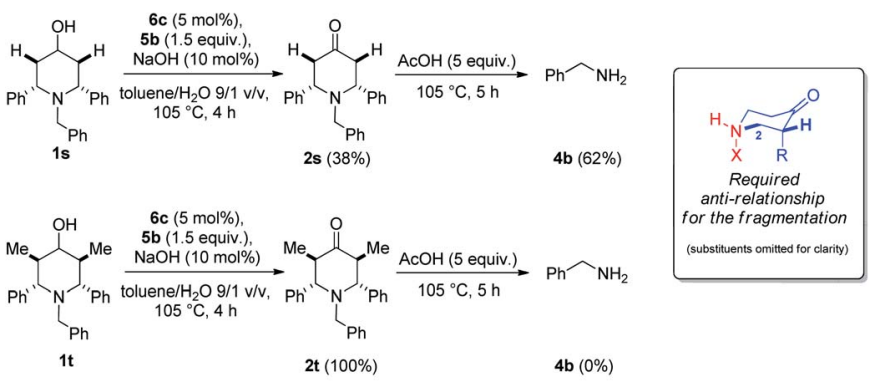

Scheme 5 Complementary experiments.

amine 4q although in lower yield (38\%). Interestingly, the method could be utilized on a large scale (4 grams) as $\mathbf{4 b}$ was obtained in $86 \%$ yield through simple acid-base extraction. In addition, treatment of the organic phase with ammonia (14 M in $\mathrm{MeOH}$ ) allowed the recovery of 2,2,6,6-tetramethylpiperidin4-one 2 in quantitative yield. ${ }^{30}$

It should be stressed that amino-ester (see 1r) could be directly involved in a three steps one pot process oxidationfragmentation-cyclization reaction to give the corresponding pure lactam 7 in $98 \%$ isolated yield without further purification (Scheme 4).

Complementary experiments were performed in order to elucidate the oxidative fragmentation mechanism of piperidinone 2 into primary amines 4. A $\mathrm{E}_{1 \mathrm{C}} \mathrm{B}$ type mechanism involving the enolate form of product 2 can be ruled out because this ring opening process was unsuccessful under basic conditions (Table 2, entry 1 ) and required the addition of acetic acid to occur (Table 2, entries 5-7). In order to get insights into this mechanism, we decided to compare the reactivity of two different substrates $N$-benzyl-2,6-diphenylpiperidin-4-ol 1s and $N$-benzyl-2,6-dimethyl-3,5-diphenylpiperidin-4-ol 1t. After reactions under our optimized conditions 1s gave a mixture of $2 \mathbf{s}: \mathbf{4 b}$ in a $38: 62$ ratio while the reaction involving $\mathbf{1 t}$ stopped

Table 3 Control experiments to elucidate the role of the metal in the fragmentation step $^{a}$

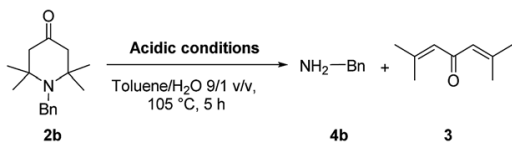

\begin{tabular}{llll}
\hline Entry & Added acid & Equiv. & $3^{b}(\%)$ \\
\hline 1 & $\mathrm{HCl}$ & 1 & - \\
2 & $\mathrm{AcOH}$ & 1 & - \\
3 & $\mathrm{BF}_{3} \cdot \mathrm{Et}_{2} \mathrm{O}$ & 0.05 & $<5$ \\
4 & $\mathrm{BF}_{3} \cdot \mathrm{Et}_{2} \mathrm{O}$ & 1 & $>90$ \\
5 & $\mathrm{Sc}^{(\mathrm{OTf})_{3}}$ & 1 & 29 \\
6 & $\mathrm{PtCl}$ & - \\
7 & {$[\mathrm{PtCl}(\mathrm{dppp})]^{+}$} & 0.05 & $<5$ \\
8 & $\mathbf{6 c}$ & 0.05 & $>90$
\end{tabular}

${ }^{a}$ All reactions were carried out with $1 \mathrm{mmol}$ of $2 \mathbf{b}$ in toluene/ $\mathrm{H}_{2} \mathrm{O} 9 / 1$ at $105{ }^{\circ} \mathrm{C} .{ }^{b}$ Determined by ${ }^{1} \mathrm{H}$ NMR spectroscopy. 


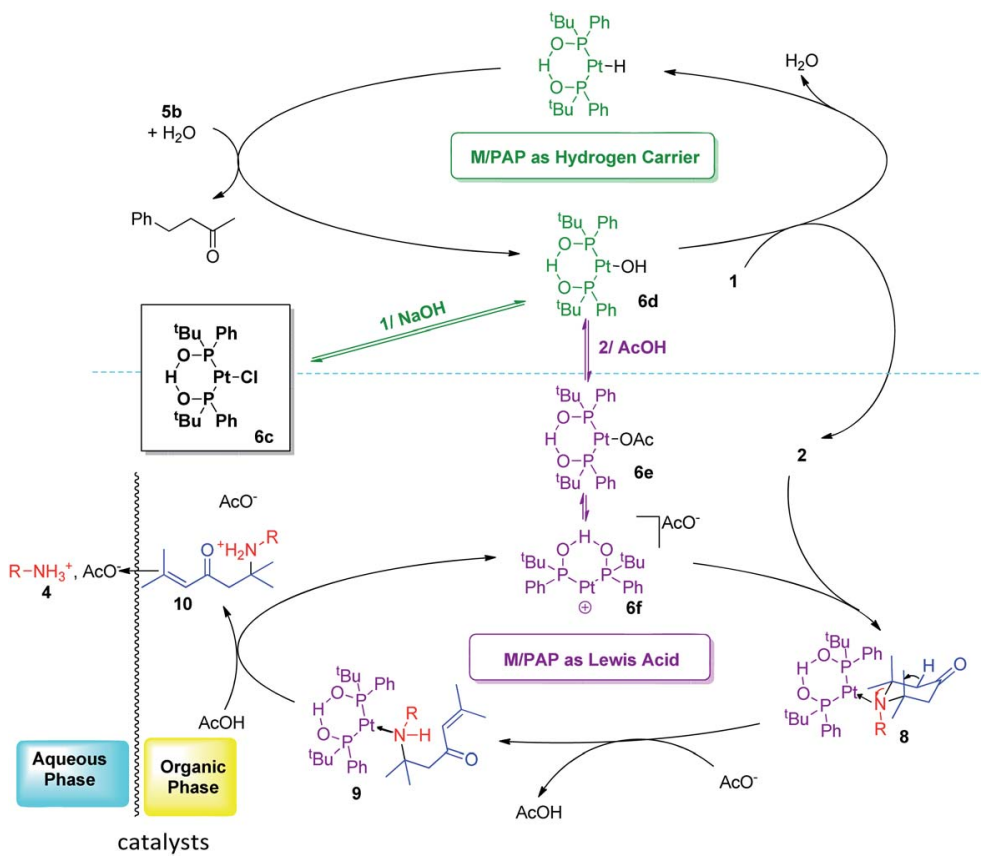

Scheme 6 Plausible pathway for cascade oxidation fragmentation by M/PAP catalysts.

at the oxidized product $2 \mathbf{t}$ (Scheme 5 ) ${ }^{31}$ Since $2 \mathrm{t}$ is present as a single diastereomer, in which the methyl and phenyl substituents are all in equatorial position the absence of fragmentation suggests the requirement of an anti-relationship between the $\mathrm{H}$ at $\mathrm{C} 2$ and the nitrogen atom (Scheme 5). ${ }^{32}$

For these reasons, we suggest that an Hofmann type degradation could proceed via the anti-deprotonation of an aminoplatinum complex. ${ }^{33,34}$ At this stage of the study, elucidating the role of the metal in the fragmentation has been found to be crucial. M/PAP moiety has been chosen because of its supposed Lewis acid activity. ${ }^{19}$ To support this assumption, reactions with $\mathbf{2 b}$ were performed in an acidic medium adding either Brønsted or Lewis acid (Table 3).

AcOH or $\mathrm{HCl}$ (1 equiv.) were inefficient for the aminone $\mathrm{C}-\mathrm{N}$ bond cleavage process (Table 3, entries 1 and 2). A stoichiometric amount of $\mathrm{BF}_{3} \cdot \mathrm{Et}_{2} \mathrm{O}$ allowed a complete conversion (Table 3, entry 4). However, a catalytic amount of $\mathrm{BF}_{3} \cdot \mathrm{Et}_{2} \mathrm{O}$ led to a low conversion (Table 3, entry 3 ). The produced primary amine probably easily coordinates $\mathrm{BF}_{3}$ thus rendering it ineffective for further ring opening. As a result, the yield of amine matches the amount of $\mathrm{BF}_{3}$ used (5\%). This also maintains the amine in the organic phase and we were unable to isolate it in pure form. Other Lewis acids such as scandium triflate (Table 3, entry 5) gave poorer results even when used in stoichiometric quantities. $\mathrm{PtCl}_{2}$ also known as a soft Lewis ${ }^{35}$ acid did not gave a better result (3 was not detected) (Table 3, entry 6). A cationic platinum complex $[\mathrm{PtCl}(\mathrm{dppp})]\left[\mathrm{PF}_{6}\right]$, in which the covalent dppp ligand mimic the supramolecular bisphosphinite chelate structure, was also inefficient (Table 3, entry 7). All these results tend to show that the PAP ligand provides the right Lewis acid character to our platinum catalyst $\mathbf{6 c}$ (Table 3 , entry 8 ) which is consistent with a Hofmann type fragmentation.
According to these results, a possible pathway for the sequence is proposed in Scheme 6. First, in basic conditions $(\mathrm{NaOH}$, Scheme 6, 1$)$, the Pt/PAP catalyst acted as a hydrogen carrier to promote the oxidation of $\mathbf{1}$ to 2 according to our previously reported mechanism..$^{18}$ Thereafter, a one pot minor modification of the experimental conditions $\mathrm{AcOH}$, Scheme 6, 2) switched Pt/PAP's properties and triggered the ring opening process. A mechanism involving a $\mathrm{Pt}(\mathrm{II})$ cationic species $6 \mathbf{f}$ playing the role of Lewis acid is suggested and can be compared to a Hofmann degradation. ${ }^{34}$ It appeared that the combined effect of both Lewis and Brønsted acid is crucial for the reaction outcome. The pathway can be described as follows: (i) the nitrogen atom coordinates to the Lewis acid (complex 8), thus promoting the ring opening (complex 9), (ii) the Brønsted acid allows the Pt-N cleavage and the resulting protonated amine $\mathbf{4}$ is carried away in the aqueous layer. In this phase transfer mechanism, the bi-talented nature of the Pt/PAP structure was clearly highlighted.

\section{Conclusions}

M/PAP complexes can be used as unique catalyst to carry out oxidation of $N$-alkyl-2,2,6,6-tetramethylpiperidin-4-ols 1 through hydrogen transfer methodology followed by $\mathrm{C}\left(\mathrm{sp}^{3}\right)-\mathrm{N}$ bond cleavage in a single operation. The self-assembled negatively charged structure of the ligand may ease the abstracting hydrogen step from 1 while the metal cationic centre may promote the formation of amino-platinum complex species from 2 responsible fora Hofmann-type degradation so as to afford a primary amine 4. As $N$-alkyl-2,2,6,6tetramethylpiperidin-4-ols are easily accessible from the cheap 2,2,6,6-tetramethylpiperidin-4-ol, ${ }^{28}$ this cascade reaction 
sequence provides an interesting entry to aliphatic primary amines merely isolated after simple acid-base extraction. Finally, the generated phorone 3 could readily be recycled into 2,2,6,6-tetramethylpiperidin-4-ol by reacting with ammonia and the method is practicable on a large scale (see ESI $\dagger$ ). The proposed mechanism for this sequence clearly shows the multiskilling aspect of M/PAP catalysts.

\section{Conflicts of interest}

There are no conflicts to declare.

\section{Acknowledgements}

This work was supported by Ministère de l'Enseignement Supérieur et de la Recherche (R. Membrat PhD grant). The authors want to thank Dr. Valérie Monnier and Dr Christophe Chendo for ESI-MS experiments and Dr Alphonse Tenaglia for fruitful discussions. Umicore AG \& Co. KG is acknowledged for the generous gift of platinum complexes.

\section{Notes and references}

1 J. Muzart, Eur. J. Org. Chem., 2015, 2015, 5693-5707.

2 D. Wang and D. Astruc, Chem. Rev., 2015, 115, 6621-6686.

3 B. Karimi and A. Zamani, J. Iran. Chem. Soc., 2008, 5, S1-S20. 4 D. Wang, A. B. Weinstein, P. B. White and S. S. Stahl, Chem. Rev., 2018, 118, 2636-2679.

5 J. Muzart, Tetrahedron, 2003, 59, 5789-5816.

6 B. Ding, Z. Zhang, Y. Liu, M. Sugiya, T. Imamoto and W. Zhang, Org. Lett., 2013, 15, 3690-3693.

7 Y. Tsuchiya, Y. Hamashima and M. Sodeoka, Org. Lett., 2006, 8, 4851-4854.

8 K. M. Gligorich, S. A. Cummings and M. S. Sigman, J. Am. Chem. Soc., 2007, 129, 14193-14195.

9 S. M. Podhajsky, Y. Iwai, A. Cook-Sneathen and M. S. Sigman, Tetrahedron, 2011, 67, 4435-4441.

10 H. Hikawa, T. Koike, K. Izumi, S. Kikkawa and I. Azumaya, Adv. Synth. Catal., 2016, 358, 784-791.

11 S. M. Podhajsky and M. S. Sigman, Organometallics, 2007, 26, 5680-5686.

12 M. J. Spallek, S. Stockinger, R. Goddard and O. Trapp, Adv. Synth. Catal., 2012, 354, 1466-1480.

13 O. Kose and S. Saito, Org. Biomol. Chem., 2010, 8, 896-900.

14 A. Martínez-Asencio, M. Yus and D. J. Ramón, Synthesis, 2011, 2011, 3730-3740.
15 G.-J. ten Brink, I. W. C. E. Arends and R. A. Sheldon, Adv. Synth. Catal., 2002, 344, 355-369.

16 A. Vasseur, R. Membrat, D. Gatineau, A. Tenaglia, D. Nuel and L. Giordano, ChemCatChem, 2017, 9, 728-732.

17 P. M. Castro, H. Gulyás, J. Benet-Buchholz, C. Bo, Z. Freixa and P. W. N. M. van Leeuwen, Catal. Sci. Technol., 2011, 1, 401.

18 R. Membrat, A. Vasseur, A. Martinez, L. Giordano and D. Nuel, Eur. J. Org. Chem., 2018, 2018, 5427-5434.

19 J. Bigeault, I. de Riggi, Y. Gimbert, L. Giordano and G. Buono, Synlett, 2008, 2008, 1071-1075.

20 T. Achard, CHIMIA International Journal for Chemistry, 2016, 70, 8-19.

21 J.-Y. Laronze, J. Sapi and J. Lévy, Synthesis, 1988, 1988, 619621.

22 J. Lévy, J.-Y. Laronze and J. Sapi, Tetrahedron Lett., 1988, 29, 3303-3306.

23 P. Aschwanden, C. R. J. Stephenson and E. M. Carreira, Org. Lett., 2006, 8, 2437-2440.

24 M. Shimano and A. I. Meyers, J. Org. Chem., 1995, 60, 74457455.

25 H. Shi, D. J. Babinski and T. Ritter, J. Am. Chem. Soc., 2015, 137, 3775-3778.

26 H. Seo, M. H. Katcher and T. F. Jamison, Nat. Chem., 2017, 9, 453-456.

27 1,4 Addition products and polymers were detected by ${ }^{1} \mathrm{H}$ NMR spectroscopy and low resolution ESIMS experiments.

28 R. Membrat, A. Vasseur, L. Giordano, A. Martinez and D. Nuel, Tetrahedron Lett., 2019, 60, 240-243.

29 For aliphatic electrophiles, see the procedure in the ESI (Section 2.3†).

30 For procedure and yield see ESI (Section $2.5 \dagger$ ).

31 The fact that $\mathbf{1 s}$ is less reactive than the other $\mathbf{1}$ substrates clearly stress that the presence of the two methyl groups in $\alpha$ position due to the nitrogen atom is crucial for the ring opening to occur (see ref. 34 ).

32 T. Ravindran, R. Jeyaraman, R. W. Murray and M. Singh, J. Org. Chem., 1991, 56, 4833-4840.

33 A. M. Belostotskii and A. B. Shapiro, Chem. Heterocycl. Compd., 1984, 20, 761-766.

34 A. M. Belostotskii and A. B. Shapiro, Chem. Heterocycl. Compd., 1987, 23, 665-669.

35 S. Kobayashi, T. Busujima and S. Nagayama, Chem.-Eur. J., 2000, 6, 3491-3494. 